\title{
Une avulsion traumatique d'un fléchisseur commun profond d'un doigt
}

\section{Traumatic Avulsion of Flexor Digitorum Profundus}

\author{
P. Kauffmann $\cdot$ C. Renfer $\cdot$ P. Le Borgne \\ Reçu le 21 août 2015 ; accepté le 25 septembre 2015 \\ (C) SFMU et Lavoisier SAS 2015
}

Un homme de 36 ans s'est présenté aux urgences suite à un traumatisme de la main droite au cours d'un match de football. Le patient ne présente aucun antécédent notable. L'examen clinique initial retrouve une ecchymose de la pulpe du $3^{\text {e }}$ doigt avec impossibilité de fléchir activement la phalange distale. La palpation du doigt est douloureuse sur l'ensemble de la face palmaire, particulièrement en regard de l'articulation interphalangienne distale (IPD). Le reste de l'examen est normal. La radiographie retrouve une fissure transversale de la phalange distale et un arrachement osseux palmaire qui correspond à l'avulsion de l'insertion distale du tendon du fléchisseur commun profond (Fig. 1). Cette lésion, également connue sous le nom de « rugby finger» ou « jersey finger », est rare. Elle fut initialement décrite en 1960 chez le rugbyman [1]. Elle se produit classiquement lors de la tentative d'agrippement d'un maillot dans les sports de contact par flexion contrariée du bout d'un doigt; le $4^{\mathrm{e}}$ doigt est le plus souvent lésé parce qu'il est le plus long lors de la préhension. Un déficit de la flexion active de l'IPD doit faire systématiquement rechercher cette atteinte mais la douleur initiale aspécifique et la rareté de la lésion rendent le diagnostic difficile pour le clinicien. L'avulsion distale de ce tendon s'accompagne presque toujours d'un fragment osseux, parfois discret, qu'il convient de savoir repérer sur le cliché de profil. Le déplacement de ce fragment est habituellement modéré ; il vient se bloquer en regard de l'IPD comme ici, ou en regard de la $1^{\text {ère }}$ phalange sous la décussation du fléchisseur commun superficiel, mais il peut exceptionnellement migrer jusqu'à la paume. En cas de difficulté, l'échographie peut permettre d'asseoir le diagnostic [2]. Le pronostic est

P. Kauffmann · C. Renfer · P. Le Borgne $(\square)$

Service d'accueil des urgences, CHU de Strasbourg,

Hôpital de Hautepierre, 1 avenue Molière,

F-67098 Strasbourg, France

e-mail : pierrick_med@yahoo.fr

P. Le Borgne

Faculté de médecine, université de Strasbourg,

4 rue Kirschleger, F-67000 Strasbourg

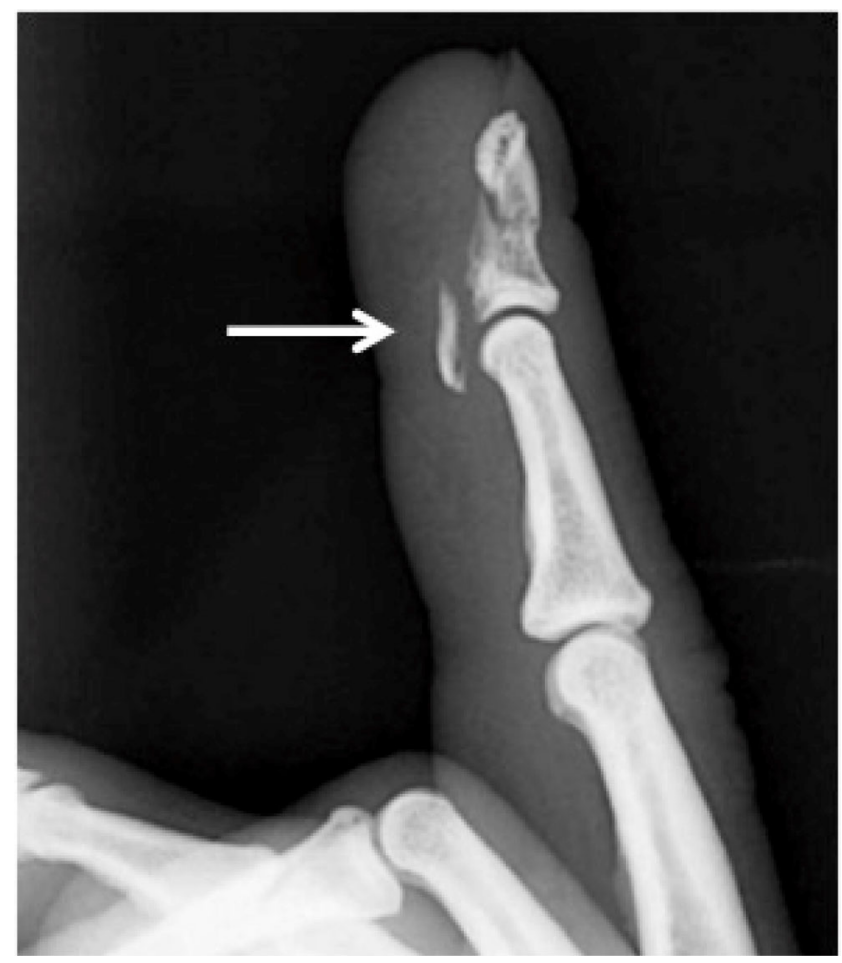

Fig. 1 Radiographie standard du $3^{\mathrm{e}}$ doigt, profil. Flèche : arrachement osseux (face palmaire) en regard de l'articulation interphalangienne distale

fonctionnel, potentiellement grave quand la lésion est passée inaperçue. Le traitement doit être rapidement chirurgical. Il a consisté pour notre patient en une réinsertion tendineuse trans-osseuse en urgence.

\section{Références}

1. Gunter J (1960) Traumatic avulsion of the insertion of flexor digitorum profundus. Aust NZ J Surg 30:1-9

2. de Gautard G, de Gautard R, Celi J, et al (2009) Sonography of jersey finger. J Ultrasound Med 28:389-92 